\title{
Instanton bound states in ABJM theory
}

\author{
Yasuyuki Hatsuda, $^{a, b}$ Sanefumi Moriyama ${ }^{c}$ and Kazumi Okuyama ${ }^{d}$ \\ ${ }^{a}$ DESY Theory Group, DESY Hamburg, \\ Notkestrasse 85, D-22603 Hamburg, Germany \\ ${ }^{b}$ Department of Physics, Tokyo Institute of Technology, \\ Tokyo 152-8551, Japan \\ ${ }^{c}$ Kobayashi Maskawa Institute $\mathcal{E}$ Graduate School of Mathematics, \\ Nagoya University, Nagoya 464-8602, Japan \\ ${ }^{d}$ Department of Physics, Shinshu University, \\ Matsumoto 390-8621, Japan \\ E-mail: yasuyuki.hatsuda@desy.de, moriyama@math.nagoya-u.ac.jp, \\ kazumi@azusa.shinshu-u.ac.jp
}

ABSTRACT: The partition function of the ABJM theory receives non-perturbative corrections due to instanton effects. We study these non-perturbative corrections, including bound states of worldsheet instantons and membrane instantons, in the Fermi-gas approach. We require that the total non-perturbative correction should be always finite for arbitrary Chern-Simons level. This finiteness is realized quite non-trivially because each bound state contribution naively diverges at some levels. The poles of each contribution should be canceled out in total. We use this pole cancellation mechanism to find unknown bound state corrections from known ones. We conjecture a general expression of the bound state contribution. Summing up all the bound state contributions, we find that the effect of bound states is simply incorporated into the worldsheet instanton correction by a redefinition of the chemical potential in the Fermi-gas system. Analytic expressions of the 3and 4-membrane instanton corrections are also proposed.

Keywords: Matrix Models, Chern-Simons Theories, Nonperturbative Effects, M-Theory

ArXiv EPrint: 1301.5184 


\section{Contents}

1 Introduction 1

2 Review of the Fermi-gas approach 4

2.1 Fermi-gas formalism 4

2.2 Non-perturbative corrections to the grand potential 5

$\begin{array}{lll}3 & \text { Bound state corrections } & \mathbf{7}\end{array}$

$3.1(1, n)$ bound state $\quad 7$

$3.2(2, n)$ and $(3, n)$ bound states $\quad 9$

$\begin{array}{lll}3.3 & \text { Summing up all the bound states } & 10\end{array}$

4 Some observations $\quad 11$

5 Higher membrane instanton corrections $\quad 14$

$\begin{array}{lll}5.1 \text { Conjecture for } b_{3}(k) \text { and } c_{3}(k) & 15\end{array}$

$\begin{array}{lll}5.2 & \text { Further conjecture for } b_{4}(k) \text { and } c_{4}(k) & 17\end{array}$

$\begin{array}{llr}6 & \text { Summary and discussions } & 18\end{array}$

A $J^{(\mathrm{np})}(k, \mu)$ at $k=1,2,3,4,6 \quad 19$

B Known results of membrane instanton corrections 20

$\begin{array}{ll}\text { C Relation of } \mu \text { and } \mu_{\text {eff }} \text { for even } k & 21\end{array}$

\section{Introduction}

Recently, there has been much progress in the 3 -dimensional $\mathcal{N}=6$ supersymmetric ChernSimons-matter theory with gauge group $\mathrm{U}(N)_{k} \times \mathrm{U}(N)_{-k}$, known as the Aharony-BergmanJafferis-Maldacena (ABJM) theory [1]. Since the ABJM theory is believed to describe the low energy effective theory on the $N$ multiple M2-branes, the study of this theory is a significant step to understand M-theory. It was shown in [2-4] that the partition function and vacuum expectation values of BPS Wilson loops in 3d supersymmetric Chern-Simons theories including the ABJM theory are reduced to finite-dimensional matrix integrals after the localization technique [5] is applied. This reduction allows us to use many matrix model techniques. In [6], the famous $N^{3 / 2}$ degrees of freedom of M2-branes predicted from the AdS/CFT correspondence [7] was reproduced from the free energy of the matrix model in the leading 't Hooft expansion. After that, it was shown in [8] that the all-genus summation 
results in the expression by the Airy function if the worldsheet instanton corrections are dropped out.

In [9], another formulation to study the ABJM matrix model was introduced. The crucial point is that the ABJM partition function is regarded as the partition function of an ideal Fermi-gas system. ${ }^{1}$ This equivalence enables us to apply the standard methods in quantum mechanics and statistical mechanics. Since the Chern-Simons level $k$ plays the role of the Planck constant $\hbar=2 \pi k$, the WKB expansion corresponds to the expansion around $k=0$, which is the strong coupling limit in the ABJM theory. In [9], such a WKB expansion of the partition function was studied in detail, and further developed in [15]. The Fermi-gas formalism is also powerful to compute the partition function exactly. In $[16,17]$, the exact ABJM partition function at $k=1$ was computed up to some values of $N$. In [18], we further developed the method to compute the partition function and obtain its exact values for higher $N$ at various levels $k$. Using these exact values, we can extract the non-perturbative corrections to the partition function by subtracting the perturbative Airy function contribution. The result is recapitulated in (A.1), with the notation explained below.

In the Fermi-gas formalism, it is useful to consider the grand partition function and the grand potential. The grand potential is divided into the following three contributions,

$$
J(k, \mu)=J^{(\text {pert })}(k, \mu)+J^{(\mathrm{np})}(k, \mu)+J^{(\mathrm{osc})}(k, \mu),
$$

where $J^{(\text {pert })}(k, \mu), \quad J^{(\mathrm{np})}(k, \mu)$ and $J^{(\mathrm{osc})}(k, \mu)$ represent the perturbative, the nonperturbative and the oscillatory parts, respectively. The oscillatory part $J^{\text {(osc) }}(k, \mu)$ arises because the perturbative and the non-perturbative parts do not respect the periodicity in $\mu$ that the grand partition function originally has. However, as discussed in [18], this oscillatory part can be removed by the deformation of the contour when we consider the partition function. Thus we neglect it in this paper. The perturbative part was computed in $[9]$ and is given by

$$
J^{(\text {pert })}(k, \mu)=\frac{C(k)}{3} \mu^{3}+B(k) \mu+A(k)
$$

where

$$
B(k)=\frac{k}{24}+\frac{1}{3 k}, \quad C(k)=\frac{2}{\pi^{2} k} .
$$

The small $k$ expansion of $A(k)$ was first computed in [9], and then its exact form was proposed in [19]. Using this result, one can easily rederive the perturbative partition function including all the $1 / N$ corrections [8].

In the present paper, we study the non-perturbative part in more detail. It is known that there are at least two kinds of instantons that induce the non-perturbative corrections in the ABJM theory. One is called the worldsheet instanton, and the other the membrane

\footnotetext{
${ }^{1}$ Various ideas related to this formulation were proposed, including the mirror expression in $[2,10]$, generalizations to less supersymmetric case [11] or Wilson loop expectation values [12], and studies [13] of the ABJ partition functions [14].
} 
instanton. From the viewpoint of the Type IIA string theory on $A d S_{4} \times \mathbb{C P}^{3}$, which is holographically dual to the ABJM theory in the 't Hooft limit, the worldsheet instanton comes from the fundamental string wrapping the holomorphic cycle $\mathbb{C P}^{1} \subset \mathbb{C P}^{3}[6,20]$, while the membrane instanton comes from the D2-brane wrapping the Lagrangian submanifold $\mathbb{R P}^{3} \subset \mathbb{C P}^{3}[21]$. The membrane instanton correction and the worldsheet instanton correction to the grand potential take the following forms [9]:

$$
J^{\mathrm{M} 2}(k, \mu)=\sum_{\ell=1}^{\infty}\left[a_{\ell}(k) \mu^{2}+b_{\ell}(k) \mu+c_{\ell}(k)\right] e^{-2 \ell \mu}, \quad J^{\mathrm{WS}}(k, \mu)=\sum_{m=1}^{\infty} d_{m}(k) e^{-\frac{4 m \mu}{k}} .
$$

When $k \ll N^{1 / 5}$, it is more appropriate to consider the M-theory on $A d S_{4} \times S^{7} / \mathbb{Z}_{k}$ as the holographic dual. From this M-theory perspective, the membrane instantons and the worldsheet instantons are both coming from the M2-branes wrapping some 3-cycles $\mathcal{M}$ and $\mathcal{W}$ in $S^{7} / \mathbb{Z}_{k}$, where $\mathcal{M}$ and $\mathcal{W}$ descend to $\mathbb{R P}^{3}$ and $\mathbb{C P}^{1}$ in the Type IIA picture, respectively. As suggested in [18] and further explained in [15], in addition to such two kinds of instantons, it is natural to expect that there is a bound state of $\ell$-membrane instanton and $m$-worldsheet instanton which gives rise to the correction of order $\mathcal{O}\left(e^{-\left(2 \ell+\frac{4 m}{k}\right) \mu}\right)$. In the M-theory picture, such an $(\ell, m)$ bound state corresponds to an M2-brane wrapping $\ell$ times on $\mathcal{M}$ and $m$ times on $\mathcal{W}$. Therefore the non-perturbative part is expected to have the following form,

$$
J^{(\mathrm{np})}(k, \mu)=\sum_{\substack{\ell, m=0 \\(\ell, m) \neq(0,0)}}^{\infty} f_{\ell, m}(k, \mu) \exp \left[-\left(2 \ell+\frac{4 m}{k}\right) \mu\right],
$$

where the membrane instanton correction corresponds to $m=0$, while the worldsheet instanton correction to $\ell=0$.

Our goal is to determine the coefficient $f_{\ell, m}(k, \mu)$ of the $(\ell, m)$ bound state correction. In the Fermi-gas approach, the membrane instanton corrections appear as exponentially suppressed corrections in $\mu$, while the worldsheet instanton corrections appear as quantum mechanical instantons. This means that the membrane instantons can be captured in the standard WKB analysis but the worldsheet instantons are invisible in the small $k$ expansion. Fortunately, since the worldsheet instanton corrections are related to the known results of the topological string on local $\mathbb{F}_{0}$, we can systematically compute $d_{m}(k)$, as pointed out in [18]. The bound state contributions are also invisible in the WKB analysis. Moreover, it is unclear how these corrections are understood in the topological string context. There are no results on the bound state correction $f_{\ell, m}(k, \mu)$ so far.

To overcome this difficulty, we employ the following strategy. We require that the nonperturbative correction $J^{(\mathrm{np})}(k, \mu)$ should be always finite for arbitrary value of $k$. This fact was first observed in [18] for some integers $k$. This requirement is realized very non-trivially because each bound state contribution $f_{\ell, m}(k, \mu)$ naively has poles at some values of $k$. As an example, let us consider the $\mathcal{O}\left(e^{-2 \mu}\right)$ correction at $k=2 n(n \in \mathbb{Z})$. At $k=2 n$, the $\mathcal{O}\left(e^{-2 \mu}\right)$ correction comes from the two contributions: the 1-membrane instanton and the $n$-worldsheet instanton. There are no bound state contributions at this order. As pointed 
out in [18], the $n$-worldsheet instanton contribution $f_{0, n}(k, \mu)$ diverges at $k=2 n$. Thus the 1-membrane instanton contribution $f_{1,0}(k, \mu)$ must also diverge at $k=2 n$, and the sum of these two contributions becomes finite. Using this pole cancellation mechanism in addition to the matching of a few coefficients of the WKB expansion, we proposed in [18] the explicit form of the 1-membrane instanton correction $f_{1,0}(k, \mu)$. Recently, the 2-membrane instanton contribution $f_{2,0}(k, \mu)$ was also proposed in [15] using a similar strategy. Here we use the pole cancellation mechanism to find the general bound state correction $f_{\ell, m}(k, \mu)$.

Remarkably, from this pole cancellation mechanism and some other input data, we can conjecture the explicit form of $f_{\ell, m}(k, \mu)$. Our conjecture resolves all the discrepancies observed in our previous paper [18]. After summing up all the bound state contributions, we find that the bound state contributions are incorporated into the worldsheet instanton correction by a simple redefinition of the chemical potential,

$$
J^{(\mathrm{np})}(k, \mu)=J^{\mathrm{M} 2}(k, \mu)+J^{\mathrm{WS}}\left(k, \mu_{\mathrm{eff}}\right),
$$

where the redefined chemical potential $\mu_{\mathrm{eff}}$ is given by

$$
\mu_{\mathrm{eff}}=\mu+\frac{1}{C(k)} \sum_{\ell=1}^{\infty} a_{\ell}(k) e^{-2 \ell \mu}
$$

Thus our remaining task is to determine the membrane instanton correction $J^{\mathrm{M} 2}(k, \mu)$ and the worldsheet instanton correction $J^{\mathrm{WS}}(k, \mu)$. As mentioned above, $J^{\mathrm{WS}}(k, \mu)$ can be systematically computed up to any desired order once the diagonal Gopakumar-Vafa (GV) invariants on local $\mathbb{F}_{0}$ is known. The membrane instanton correction $J^{\mathrm{M} 2}(k, \mu)$ will be also determined order by order. In this paper, we further propose the 3 - and 4-membrane instanton corrections explicitly.

The organization of this paper is as follows. In the next section, we shall first review the Fermi gas formalism. In section 3 we shall embark on our study of the bound state corrections. In section 4, we make several observations about the general structure of membrane instanton corrections. Using the structure found in section 4 , in section 5 we proceed to higher membrane instanton corrections. Finally we conclude in section 6. Appendices $\mathrm{A}$ and $\mathrm{B}$ are devoted to the summary of the known results of the nonperturbative corrections of grand potential and the instanton coefficients. In appendix C,

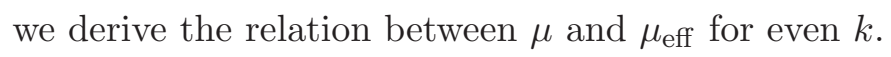

\section{Review of the Fermi-gas approach}

Let us start by reviewing the Fermi-gas formalism describing the ABJM partition function proposed in [9] and the non-perturbative corrections to the grand potential $[9,15,18]$.

\subsection{Fermi-gas formalism}

The partition function of ABJM theory on a round $S^{3}$ is written as a matrix integral [2]

$$
Z(k, N)=\frac{1}{(N !)^{2}} \int \frac{d^{N} \mu_{i}}{(2 \pi)^{N}} \frac{d^{N} \nu_{i}}{(2 \pi)^{N}} \frac{\prod_{i<j}\left[2 \sinh \frac{\mu_{i}-\mu_{j}}{2}\right]^{2}\left[2 \sinh \frac{\nu_{i}-\nu_{j}}{2}\right]^{2}}{\prod_{i, j}\left[2 \cosh \frac{\mu_{i}-\nu_{j}}{2}\right]^{2}} \exp \left[\frac{i k}{4 \pi} \sum_{i}\left(\mu_{i}^{2}-\nu_{i}^{2}\right)\right] .
$$


The key observation in [9] is that $(2.1)$ can be recast into the partition function of a Fermi-gas system

$$
Z(k, N)=\frac{1}{N !} \sum_{\sigma \in S_{N}}(-1)^{\epsilon(\sigma)} \int \frac{d^{N} q}{(2 \pi)^{N}} \prod_{i} \rho\left(q_{i}, q_{\sigma(i)}\right),
$$

with the density matrix given by

$$
\rho\left(q_{1}, q_{2}\right)=\frac{1}{k} \frac{1}{\sqrt{2 \cosh \frac{q_{1}}{2}}} \frac{1}{2 \cosh \frac{q_{1}-q_{2}}{2 k}} \frac{1}{\sqrt{2 \cosh \frac{q_{2}}{2}}} .
$$

After introducing the chemical potential $\mu$ or the fugacity $z=e^{\mu}$, the grand partition function

$$
\Xi(k, \mu)=1+\sum_{N=1}^{\infty} Z(k, N) e^{\mu N}
$$

is written as the well-known form for the Fermi-gas system,

$$
\Xi(k, \mu)=\prod_{j=0}^{\infty}\left(1+e^{-\left(E_{j}-\mu\right)}\right),
$$

where $E_{j}$ is the energy spectrum of the one-particle Hamiltonian defined by

$$
\hat{\rho}=e^{-\hat{H}}, \quad\left\langle q_{1}|\hat{\rho}| q_{2}\right\rangle=\rho\left(q_{1}, q_{2}\right) .
$$

Mathematically, the grand partition function is nicely expressed as a Fredholm determinant for the kernel $\rho$,

$$
\Xi(k, \mu)=\operatorname{det}(1+z \rho)=\exp \left[-\sum_{n=1}^{\infty} \frac{(-z)^{n}}{n} \operatorname{Tr} \rho^{n}\right] .
$$

We are interested in the non-perturbative corrections to the grand potential defined by

$$
J(k, \mu)=\log \Xi(k, \mu) .
$$

Note that the partition function can be reconstructed from the grand partition function by

$$
Z(k, N)=\oint \frac{d z}{2 \pi i} \frac{\Xi(k, \mu)}{z^{N+1}}=\int_{-\pi i}^{\pi i} \frac{d \mu}{2 \pi i} e^{J(k, \mu)-N \mu} .
$$

\subsection{Non-perturbative corrections to the grand potential}

As mentioned in the introduction, the grand potential receives non-perturbative corrections. The worldsheet instanton correction $J^{\mathrm{WS}}(k, \mu)$ can be systematically computed by using the results of the topological string on local $\mathbb{F}_{0}$,

$$
J^{\mathrm{WS}}(k, \mu)=\sum_{g=0}^{\infty} \sum_{n, d=1}^{\infty} n_{d}^{g}\left(2 \sin \frac{2 \pi n}{k}\right)^{2 g-2} \frac{(-1)^{d n}}{n} e^{-\frac{4 d n \mu}{k}},
$$




\begin{tabular}{|cccccccc|}
\hline$d$ & 1 & 2 & 3 & 4 & 5 & 6 & 7 \\
\hline$n_{d}^{0}$ & -4 & -4 & -12 & -48 & -240 & -1356 & -8428 \\
$n_{d}^{1}$ & 0 & 0 & 0 & 9 & 136 & 1616 & 17560 \\
$n_{d}^{2}$ & 0 & 0 & 0 & 0 & -24 & -812 & -17340 \\
$n_{d}^{3}$ & 0 & 0 & 0 & 0 & 0 & 186 & 9712 \\
$n_{d}^{4}$ & 0 & 0 & 0 & 0 & 0 & -16 & -3156 \\
$n_{d}^{5}$ & 0 & 0 & 0 & 0 & 0 & 0 & 552 \\
$n_{d}^{6}$ & 0 & 0 & 0 & 0 & 0 & 0 & -40 \\
$n_{d}^{7}$ & 0 & 0 & 0 & 0 & 0 & 0 & 0 \\
\hline
\end{tabular}

Table 1. The diagonal GV invariants on local $\mathbb{F}_{0}$.

where $n_{d}^{g}$ denotes the diagonal GV invariants on local $\mathbb{F}_{0}[22]$

$$
n_{d}^{g}=\sum_{d_{1}+d_{2}=d} n_{d_{1}, d_{2}}^{g} .
$$

It is easy to see that the coefficient $d_{m}(k)$ is explicitly written as

$$
d_{m}(k)=\sum_{g=0}^{\infty} \sum_{d \mid m} n_{d}^{g} \frac{(-1)^{m} d}{m}\left(2 \sin \frac{2 \pi m}{d k}\right)^{2 g-2},
$$

where $d \mid m$ means that $d$ is a divisor of $m$. Using the data of GV invariants in $[22,23]$, we can compute $n_{d}^{g}$ up to $(g, d)=(7,7)$. The result is summarized in table 1 . From these values, we can compute $d_{m}(k)$ up to $m=7$.

It is more difficult to determine the membrane instanton contribution $f_{\ell, 0}(k, \mu)=$ $a_{\ell}(k) \mu^{2}+b_{\ell}(k) \mu+c_{\ell}(k)$. In $[9,15]$, the WKB expansions of the coefficients $a_{\ell}(k), b_{\ell}(k)$ and $c_{\ell}(k)$ were studied. In [18], the 1-membrane instanton correction $f_{1,0}(k, \mu)$ was proposed based on the requirement of the pole cancellation and the matching of the small $k$ expansion. Recently, the 2-membrane instanton correction $f_{2,0}(k, \mu)$ was also proposed in a similar way [15]. The results are summarized in (B.1), (B.2), and (B.3).

Let us here sketch the pole cancellation mechanism, which is important in our later analysis. We first notice that the coefficients (2.12), (B.1) and (B.2) have poles at some values of $k$. The crucial point is that these poles should be canceled by other contributions including the bound states at the same order. The final result of $J^{(\mathrm{np})}(k, \mu)$ is finite even for these values of $k$. As an example, let us consider the $\mathcal{O}\left(e^{-2 \mu}\right)$ corrections for even integer $k=2 n$. In this case there are two contributions: the 1-membrane instanton and the $n$-worldsheet instanton. At this order, there are no bound states, and the total nonperturbative correction is given by the sum of the 1-membrane instanton correction and the $n$-worldsheet instanton correction. At $k=2 n$, the coefficients $b_{1}(k)$ and $c_{1}(k)$ are divergent. One can easily check that the behavior near $k=2 n$ is given by

$$
\begin{aligned}
\lim _{k \rightarrow 2 n} a_{1}(k) & =(-1)^{n-1} \frac{2}{n \pi^{2}}, \\
\lim _{k \rightarrow 2 n} b_{1}(k) & =(-1)^{n} \frac{4}{\pi^{2}(k-2 n)},
\end{aligned}
$$




$$
\lim _{k \rightarrow 2 n} c_{1}(k)=(-1)^{n-1}\left[-\frac{4 n}{\pi^{2}(k-2 n)^{2}}-\frac{4}{\pi^{2}(k-2 n)}+\frac{1}{3}\left(\frac{1}{n}-2 n\right)\right],
$$

On the other hand, at $k=2 n$, the worldsheet instanton correction $f_{0, n}(k, \mu)$ is also divergent. Its pole structure is given by

$$
\lim _{k \rightarrow 2 n} f_{0, n} e^{-\frac{4 n}{k} \mu}=(-1)^{n-1}\left[\frac{4 n}{\pi^{2}(k-2 n)^{2}}+\frac{4(\mu+1)}{\pi^{2}(k-2 n)}+\frac{2 \mu^{2}+2 \mu+1}{n \pi^{2}}+w_{n}\right] e^{-2 \mu},
$$

where $w_{n}$ are some constants. The poles from $f_{1,0}$ and $f_{0, n}$ precisely cancel, and we get the final answer,

$$
\lim _{k \rightarrow 2 n}\left(f_{1,0} e^{-2 \mu}+f_{0, n} e^{-\frac{4 n}{k} \mu}\right)=(-1)^{n-1}\left[\frac{4 \mu^{2}+2 \mu+1}{n \pi^{2}}+s_{n}\right] e^{-2 \mu},
$$

where $s_{n}=w_{n}+\frac{1}{3 n}-\frac{2 n}{3}$.

In [15], such pole cancellation mechanism was considered at order $\mathcal{O}\left(e^{-4 \mu}\right)$ for odd integer $k$ to find the 2-membrane instanton coefficients (B.2). For such $k$, there are no bound state contributions either, and one can focus on the membrane instantons and the worldsheet instantons only. In the next section, we consider some other cases where the bound state instanton corrections exist. We use the pole cancellation mechanism to find the coefficients $f_{\ell, m}(k, \mu)$.

\section{Bound state corrections}

In this section, we consider the bound state contribution $f_{\ell, m}(k, \mu)$.

\section{$3.1 \quad(1, n)$ bound state}

Let us first consider the $(1, n)$ bound state. If we focus on the $\mathcal{O}\left(e^{-4 \mu}\right)$ term for even integer $k=2 n$, there are three contributions: the 2-membrane instanton, the $2 n$-worldsheet instanton and the $(1, n)$ bound state. Using (B.2), the behavior of the 2-membrane instanton correction at $k=2 n$ is given by

$$
\lim _{k \rightarrow 2 n} f_{2,0} e^{-4 \mu}=\left[\frac{41 n}{2 \pi^{2}(k-2 n)^{2}}+\frac{25(2 \mu+1)}{2 \pi^{2}(k-2 n)}+\frac{-9 \mu^{2}+4 \mu}{n \pi^{2}}+3\left(-\frac{1}{2 n}+\frac{10 n}{9}\right)\right] e^{-4 \mu} .
$$

One can check that the pole structure of the $2 n$-worldsheet instanton correction is also given by

$$
\lim _{k \rightarrow 2 n} f_{0,2 n} e^{-\frac{8 n}{k} \mu}=-9\left[\frac{n}{2 \pi^{2}(k-2 n)^{2}}+\frac{2 \mu+1}{2 \pi^{2}(k-2 n)}+\frac{2 \mu^{2}+\mu+1 / 4}{2 n \pi^{2}}+v_{n}\right] e^{-4 \mu},
$$

where $v_{n}$ are some constants whose first few values are given by

$$
v_{1}=\frac{1}{18}, \quad v_{2}=\frac{13}{36}, \quad v_{3}=\frac{193}{54} .
$$


Thus, the sum of these two corrections is

$$
\begin{aligned}
& \lim _{k \rightarrow 2 n}\left(f_{2,0} e^{-4 \mu}+f_{0,2 n} e^{-\frac{8 n}{k} \mu}\right)= {\left[\frac{16 n}{\pi^{2}(k-2 n)^{2}}+\frac{8(2 \mu+1)}{\pi^{2}(k-2 n)}-\frac{36 \mu^{2}+\mu+9 / 4}{2 n \pi^{2}}\right.} \\
&\left.+3\left(-\frac{1}{2 n}+\frac{10 n}{9}\right)-9 v_{n}\right] e^{-4 \mu} .
\end{aligned}
$$

These poles must be canceled by the contribution from the $(1, n)$ bound state.

We propose the following form of the $(1, n)$ bound state coefficient:

$$
f_{1, n}(k, \mu)=-2 n \pi^{2} a_{1}(k) d_{n}(k) .
$$

As we will see below, our proposal (3.5) passes many non-trivial tests. Firstly, (3.5) precisely cancels the poles in (3.4). Secondly, (3.5) correctly reproduces the finite part of $J^{(\mathrm{np})}(\mu)$ in (A.1). Let us see this in more detail. At $k=2 n, f_{1, n}$ in (3.5) behaves as

$$
\lim _{k \rightarrow 2 n} f_{1, n} e^{-\left(2+\frac{4 n}{k}\right) \mu}=\left[-\frac{16 n}{\pi^{2}(k-2 n)^{2}}-\frac{8(2 \mu+1)}{\pi^{2}(k-2 n)}-\frac{8 \mu^{2}}{n \pi^{2}}+u_{n}\right] e^{-4 \mu},
$$

where the first few values of the constants $u_{n}$ are given by

$$
u_{1}=\frac{2}{3}, \quad u_{2}=-\frac{2}{3}, \quad u_{3}=-\frac{94}{9} .
$$

Thus the total result becomes finite,

$$
\lim _{k \rightarrow 2 n}\left(f_{2,0} e^{-4 \mu}+f_{0,2 n} e^{-\frac{8 n}{k} \mu}+f_{1, n} e^{-\left(2+\frac{4 n}{k}\right) \mu}\right)=\left[-\frac{52 \mu^{2}+\mu / 4+9 / 16}{2 n \pi^{2}}+t_{n}\right] e^{-4 \mu},
$$

where

$$
t_{n}=3\left(-\frac{1}{2 n}+\frac{10 n}{9}\right)-9 v_{n}+u_{n}
$$

whose first three values are given by

$$
t_{1}=2, \quad t_{2}=2, \quad t_{3}=-\frac{298}{9} .
$$

These exactly agree with (A.1) for $k=2,4,6$.

Moreover, at $k=4$, the $\mathcal{O}\left(e^{-3 \mu}\right)$ terms come from $(0,3)$ and $(1,1)$ bound states. One finds

$$
\lim _{k \rightarrow 4}\left(f_{0,3} e^{-\frac{12}{k} \mu}+f_{1,1} e^{-\left(2+\frac{4}{k}\right) \mu}\right)=\left(\frac{10}{3}+2\right) e^{-3 \mu}=\frac{16}{3} e^{-3 \mu} .
$$

Similarly, at $k=6$, the $\mathcal{O}\left(e^{-8 \mu / 3}\right)$ and $\mathcal{O}\left(e^{-10 \mu / 3}\right)$ terms come from $(0,4)+(1,1)$ and $(0,5)+(1,2)$, respectively. One can easily check

$$
\begin{aligned}
& \lim _{k \rightarrow 6}\left(f_{0,4} e^{-\frac{16}{k} \mu}+f_{1,1} e^{-\left(2+\frac{4}{k}\right) \mu}\right)=\left(-8-\frac{16}{9}\right) e^{-\frac{8 \mu}{3}}=-\frac{88}{9} e^{-\frac{8 \mu}{3}} \\
& \lim _{k \rightarrow 6}\left(f_{0,5} e^{-\frac{20}{k} \mu}+f_{1,2} e^{-\left(2+\frac{8}{k}\right) \mu}\right)=\left(\frac{244}{15}+\frac{16}{3}\right) e^{-\frac{10 \mu}{3}}=\frac{108}{5} e^{-\frac{10 \mu}{3}} .
\end{aligned}
$$

All these results perfectly agree with (A.1) again.

From these checks, we strongly believe that our conjecture (3.5) is correct for any $n$. 


\section{$3.2(2, n)$ and $(3, n)$ bound states}

Next let us consider the $(2, n)$ bound states. For these bound states, we have less information. However, we here propose that $f_{2, n}(k, \mu)$ is given by the following simple and beautiful form:

$$
f_{2, n}(k, \mu)=\left[-2 n \pi^{2} a_{2}(k)+\frac{1}{2 !}\left(-2 n \pi^{2} a_{1}(k)\right)^{2}\right] d_{n}(k) .
$$

As a check of this proposal, let us consider the $\mathcal{O}\left(e^{-20 \mu / 3}\right)$ term at $k=3$. This term comes from $(0,5)$ and $(2,2)$ bound states. Thus we predict

$$
\lim _{k \rightarrow 3}\left(f_{0,5} e^{-\frac{20}{k} \mu}+f_{2,2} e^{-\left(4+\frac{8}{k}\right) \mu}\right)=\left(\frac{244}{15}+\frac{16}{3}\right) e^{-\frac{20 \mu}{3}}=\frac{108}{5} e^{-\frac{20 \mu}{3}} .
$$

Similarly, if one considers the $\mathcal{O}\left(e^{-5 \mu}\right)$ term at $k=4$ and the $\mathcal{O}\left(e^{-14 \mu / 3}\right)$ term at $k=6$, one obtain the predictions

$$
\begin{aligned}
\lim _{k \rightarrow 4}\left(f_{0,5} e^{-\frac{20}{k} \mu}+f_{1,3} e^{-\left(2+\frac{12}{k}\right) \mu}+f_{2,1} e^{-\left(4+\frac{4}{k}\right) \mu}\right) & =\left(\frac{101}{5}+20+11\right) e^{-5 \mu} \\
& =\frac{256}{5} e^{-5 \mu} \\
\lim _{k \rightarrow 6}\left(f_{0,7} e^{-\frac{28}{k} \mu}+f_{1,4} e^{-\left(2+\frac{16}{k}\right) \mu}+f_{2,1} e^{-\left(4+\frac{4}{k}\right) \mu}\right) & =\left(\frac{1712}{21}+\frac{128}{3}+\frac{248}{27}\right) e^{-\frac{14 \mu}{3}} \\
& =\frac{25208}{189} e^{-\frac{14 \mu}{3}}
\end{aligned}
$$

These correctly reproduce the result (A.1). As we will see in the next section, our proposal (3.13) is also consistent with the pole structures of $b_{3}(k)$ and $c_{3}(k)$.

From the forms of $f_{1, n}$ and $f_{2, n}$, it is natural to expect that $f_{3, n}$ takes the following form:

$$
f_{3, n}=\left[-2 n \pi^{2} a_{3}(k)+s\left(-2 n \pi^{2} a_{2}(k)\right)\left(-2 n \pi^{2} a_{1}(k)\right)+t\left(-2 n \pi^{2} a_{1}(k)\right)^{3}\right] d_{n}(k),
$$

where $s$ and $t$ are some constants. To fix $s$ and $t$, we consider the $\mathcal{O}\left(e^{-8 \mu}\right)$ terms at $k=2$. These terms come from $(4,0),(3,1),(2,2),(1,3)$ and $(0,4)$. By comparing the coefficients of $\mu^{2} e^{-8 \mu}$, we obtain the constraint

$$
-\frac{2269}{\pi^{2}}-\frac{16}{3 \pi^{2}}(50+27 s+24 t)=-\frac{2701}{\pi^{2}} .
$$

Moreover, since the $\mathcal{O}\left(e^{-7 \mu}\right)$ term at $k=4$ come from $(3,1),(2,3),(1,5)$ and $(0,7)$, we get another condition,

$$
\frac{11882}{21}+18 s+8 t=\frac{4096}{7} .
$$

From these conditions, $s$ and $t$ are completely fixed, and we find

$$
s=1, \quad t=\frac{1}{6}
$$

Thus (3.16) becomes

$$
f_{3, n}=\left[-2 n \pi^{2} a_{3}(k)+\left(-2 n \pi^{2} a_{2}(k)\right)\left(-2 n \pi^{2} a_{1}(k)\right)+\frac{1}{3 !}\left(-2 n \pi^{2} a_{1}(k)\right)^{3}\right] d_{n}(k) .
$$




\subsection{Summing up all the bound states}

Let us summarize the results in the previous subsection. For notational simplicity, we introduce

$$
A_{\ell, m} \equiv-2 m \pi^{2} a_{\ell}(k)
$$

In terms of $A_{\ell, m}$, our conjecture of the bound state contributions $f_{1, m}, f_{2, m}$ and $f_{3, m}$ in the previous subsection are written as

$$
\begin{aligned}
& f_{1, m}=A_{1, m} d_{m}, \quad f_{2, m}=\left(A_{2, m}+\frac{1}{2 !} A_{1, m}^{2}\right) d_{m}, \\
& f_{3, m}=\left(A_{3, m}+A_{2, m} A_{1, m}+\frac{1}{3 !} A_{1, m}^{3}\right) d_{m} .
\end{aligned}
$$

These expressions immediately suggest us that the general coefficient $f_{\ell, m}$ is given by

$$
f_{\ell, m}=\left[\sum_{\left(p_{1}, \ldots, p_{\ell}\right)} \frac{1}{p_{1} ! p_{2} ! \cdots p_{\ell} !} A_{1, m}^{p_{1}} A_{2, m}^{p_{2}} \cdots A_{\ell, m}^{p_{\ell}}\right] d_{m}, \quad(\ell=1,2, \ldots),
$$

where the sum runs over all the allowed partitions of $\ell$ labeled by $\left(p_{1}, \ldots, p_{\ell}\right)$ satisfying

$$
p_{1}+2 p_{2}+\cdots+\ell p_{\ell}=\ell, \quad p_{1} \geq 0, \quad p_{2} \geq 0, \cdots, \quad p_{\ell} \geq 0 .
$$

One non-trivial check of this conjecture is to predict the coefficient of $\mu^{2} e^{-10 \mu}$ at $k=2$. Using the explicit forms of $a_{\ell}(k)(\ell=1, \ldots, 5)$ in $[15]^{2}$ and our conjecture (3.23), one finds

$$
\begin{aligned}
\lim _{k \rightarrow 2} & \sum_{m=0}^{5} f_{5-m, m} e^{-\left(2(5-m)+\frac{4 m}{k}\right) \mu} \\
& =\left(\frac{31752}{5 \pi^{2}}+\frac{4150}{\pi^{2}}+\frac{5760}{\pi^{2}}+\frac{6888}{\pi^{2}}+\frac{6216}{\pi^{2}}+\frac{15002}{5 \pi^{2}}\right) \mu^{2} e^{-10 \mu}+\text { (other terms) } \\
& =\frac{161824}{5 \pi^{2}} \mu^{2} e^{-10 \mu}+\text { (other terms). }
\end{aligned}
$$

This perfectly agrees with the result in (A.1)! In the above computation, each bound state contributes quite non-trivially, and the sum of all these bound states reproduces the correct answer. Therefore this agreement strongly supports our conjecture (3.23) (at least up to $\ell=4)$.

If this conjecture is correct, we can perform the sum over $\ell$ in $J^{(\mathrm{np})}(\mu)$,

$$
\begin{aligned}
J^{(\mathrm{np})}(\mu) & =\sum_{\substack{\ell, m=0 \\
\ell, m) \neq(0,0)}}^{\infty} f_{\ell, m}(k, \mu) \exp \left[-\left(2 \ell+\frac{4 m}{k}\right) \mu\right] \\
& =\sum_{\ell=1}^{\infty} f_{\ell, 0} e^{-2 \ell \mu}+\sum_{m=1}^{\infty} d_{m} e^{-\frac{4 m}{k} \mu} \sum_{\ell=0}^{\infty} e^{-2 \ell \mu} \sum_{\left(p_{1}, \ldots, p_{\ell}\right)} \frac{1}{p_{1} ! p_{2} ! \cdots p_{\ell} !} A_{1, m}^{p_{1}} A_{2, m}^{p_{2}} \cdots A_{\ell, m}^{p_{\ell}} .
\end{aligned}
$$

\footnotetext{
${ }^{2}$ There is a typo in $a_{5}(k)$ in the first version of [15]. We need to replace $9104 \mathrm{in}$ front of $\cos (5 \pi k / 2)$ by $9104 / 5$. We are grateful to the authors of [15] for correspondence.
} 
Using the summation formula

$$
\sum_{\ell=0}^{\infty} x^{\ell} \sum_{\left(p_{1}, \ldots, p_{\ell}\right)} \frac{1}{p_{1} ! p_{2} ! \cdots p_{\ell} !} A_{1, m}^{p_{1}} A_{2, m}^{p_{2}} \cdots A_{\ell, m}^{p_{\ell}}=\exp \left[\sum_{n=1}^{\infty} x^{n} A_{n, m}\right],
$$

we finally obtain

$$
\begin{aligned}
J^{(\mathrm{np})}(\mu)=\sum_{\ell=1}^{\infty}\left(a_{\ell}(k) \mu^{2}+b_{\ell}(k) \mu+c_{\ell}(k)\right) e^{-2 \ell \mu} & \\
& +\sum_{m=1}^{\infty} d_{m}(k) \exp \left[-\frac{4 m}{k}\left(\mu+\frac{\pi^{2} k}{2} \sum_{n=1}^{\infty} a_{n}(k) e^{-2 n \mu}\right)\right] .
\end{aligned}
$$

This result means that all the bound state contributions are incorporated into the worldsheet instanton corrections by redefining the chemical potential $\mu$, as in (1.6) and (1.7).

For even $k=2 n$, in particular, we can write down the analytic relation between $\mu$ and $\mu_{\text {eff }}$,

$$
\mu_{\mathrm{eff}}=\mu+(-1)^{n-1} 2 e^{-2 \mu}{ }_{4} F_{3}\left(1,1, \frac{3}{2}, \frac{3}{2} ; 2,2,2 ;(-1)^{n} 16 e^{-2 \mu}\right) .
$$

A derivation of this expression is presented in appendix C. Similarly, for odd $k$, from the numerical value, we conjecture that

$$
\mu_{\text {eff }}=\mu+e^{-4 \mu}{ }_{4} F_{3}\left(1,1, \frac{3}{2}, \frac{3}{2} ; 2,2,2 ;-16 e^{-4 \mu}\right) .
$$

These relations are very similar to the relation between the function $\kappa$ of the 't Hooft coupling $\lambda=N / k$ and the derivative of the genus-zero free energy, found in [6].

\section{Some observations}

Concerning the forms of membrane instanton corrections, we find some curious observations. As seen in the previous section, the summation over the bound states results in the worldsheet instanton correction with the redefined chemical potential $\mu_{\text {eff }}$. This fact suggests us to rewrite the other part of the grand potential also in terms of $\mu_{\text {eff }}$. Namely, we would like to rewrite the sum of the perturbative part and the membrane instanton part

$$
\begin{aligned}
& J^{(\text {pert })}(k, \mu)+J^{\mathrm{M} 2}(k, \mu) \\
& \quad=\left[\frac{C(k)}{3} \mu^{3}+B(k) \mu+A(k)\right]+\left[\mu^{2} J_{a}(k, \mu)+\mu J_{b}(k, \mu)+J_{c}(k, \mu)\right],
\end{aligned}
$$

where we have introduced the notation

$$
J_{a}(k, \mu)=\sum_{\ell=1}^{\infty} a_{\ell}(k) e^{-2 \ell \mu}, \quad J_{b}(k, \mu)=\sum_{\ell=1}^{\infty} b_{\ell}(k) e^{-2 \ell \mu}, \quad J_{c}(k, \mu)=\sum_{\ell=1}^{\infty} c_{\ell}(k) e^{-2 \ell \mu} .
$$


When writing in terms of $\mu_{\mathrm{eff}}=\mu+J_{a}(k, \mu) / C(k)$, the $\mu^{2} J_{a}$ term in (4.1) is absorbed into the perturbative part and the final result becomes

$$
J^{(\text {pert })}(k, \mu)+J^{\mathrm{M} 2}(k, \mu)=J^{(\text {pert })}\left(k, \mu_{\mathrm{eff}}\right)+\mu_{\mathrm{eff}} \widetilde{J}_{b}\left(k, \mu_{\mathrm{eff}}\right)+\widetilde{J}_{c}\left(k, \mu_{\mathrm{eff}}\right),
$$

where $\widetilde{J}_{b}\left(k, \mu_{\text {eff }}\right)$ and $\widetilde{J}_{c}\left(k, \mu_{\text {eff }}\right)$ are given by

$$
\begin{aligned}
& \widetilde{J}_{b}\left(k, \mu_{\mathrm{eff}}\right)=\sum_{\ell=1}^{\infty} \widetilde{b}_{\ell}(k) e^{-2 \ell \mu_{\mathrm{eff}}}=J_{b}(k, \mu)-\frac{J_{a}(k, \mu)^{2}}{C(k)}, \\
& \widetilde{J}_{c}\left(k, \mu_{\mathrm{eff}}\right)=\sum_{\ell=1}^{\infty} \widetilde{c}_{\ell}(k) e^{-2 \ell \mu_{\mathrm{eff}}}=J_{c}(k, \mu)-\frac{J_{a}(k, \mu) J_{b}(k, \mu)}{C(k)}-\frac{B(k)}{C(k)} J_{a}(k, \mu)+\frac{2 J_{a}(k, \mu)^{3}}{3 C(k)^{2}} .
\end{aligned}
$$

The coefficients $\widetilde{b}_{\ell}(k)$ and $\widetilde{c}_{\ell}(k)$ are related to $a_{\ell}(k), b_{\ell}(k)$ and $c_{\ell}(k)$. The first two coefficients are explicitly found to be

$$
\begin{aligned}
& \widetilde{b}_{1}(k)=\frac{2}{\pi} \cot \left(\frac{\pi k}{2}\right) \cos \left(\frac{\pi k}{2}\right), \\
& \widetilde{b}_{2}(k)=\frac{1}{\pi} \cot (\pi k)(4+5 \cos (\pi k)),
\end{aligned}
$$

and

$$
\begin{aligned}
& \widetilde{c}_{1}(k)=\frac{1}{\pi} \cot \left(\frac{\pi k}{2}\right) \cos \left(\frac{\pi k}{2}\right)+\frac{k}{4} \csc ^{2}\left(\frac{\pi k}{2}\right) \cos \left(\frac{\pi k}{2}\right)(3-\cos (\pi k)), \\
& \widetilde{c}_{2}(k)=\frac{1}{4 \pi} \cot (\pi k)(4+5 \cos (\pi k))+\frac{k}{16} \csc ^{2}(\pi k)(16+25 \cos (\pi k)-5 \cos (3 \pi k)) .
\end{aligned}
$$

Very surprisingly, we find that $\widetilde{c}_{\ell}(k)$ can be expressed by $\widetilde{b}_{\ell}(k)$,

$$
\widetilde{c}_{\ell}(k)=-k^{2} \frac{\partial}{\partial k}\left(\frac{\widetilde{b}_{\ell}(k)}{2 \ell k}\right), \quad(\ell=1,2) .
$$

We stress that, if we use this relation, $\widetilde{c}_{\ell}(k)$ is completely determined by $\widetilde{b}_{\ell}(k)$ and subsequently the original coefficients $b_{\ell}(k)$ and $c_{\ell}(k)$ can be reconstructed by these redefined functions. Therefore we expect that the redefined function $\widetilde{b}_{\ell}(k)$ plays a more fundamental role in studying the higher membrane instanton corrections.

Furthermore, we note that $\widetilde{b}_{\ell}(k)$ has an interesting structure. Considering that the poles appearing in $d_{m}(k)$ originate from the genus-zero GV invariants $n_{d}^{0}$, we should be able to separate the divergences in $\widetilde{b}_{\ell}(k)$ and express them in terms of $n_{d}^{0}$. In fact we find that $\widetilde{b}_{\ell}(k)$ can be written as

$$
\begin{aligned}
& \widetilde{b}_{1}(k)=-\frac{1}{2 \pi} \cos \left(\frac{\pi k}{2}\right)\left[n_{1}^{0} \cot \left(\frac{\pi k}{2}\right)\right], \\
& \widetilde{b}_{2}(k)=-\frac{1}{\pi} \cos (\pi k)\left[n_{2}^{0} \cot \left(\frac{\pi k}{2}\right)+\frac{n_{1}^{0}}{2^{2}} \cot (\pi k)\right] .
\end{aligned}
$$

From this, we conjecture that the general structure of $\widetilde{b}_{\ell}(k)$ is given by

$$
\widetilde{b}_{\ell}(k)=-\frac{\ell}{2 \pi} \cos \left(\frac{\pi k \ell}{2}\right) \sum_{d \mid \ell} \frac{n_{d}^{0}}{(\ell / d)^{2}} \cot \left(\frac{\pi k \ell}{2 d}\right)+\text { (regular). }
$$


As we will see in the next section, the regular part has the following structure

$$
\widetilde{b}_{\ell}(k)=-\frac{\ell}{2 \pi} \cos \left(\frac{\pi k \ell}{2}\right)\left[\sum_{d \mid \ell} \frac{n_{d}^{0}}{(\ell / d)^{2}} \cot \left(\frac{\pi k \ell}{2 d}\right)+\sum_{n=1}^{N_{\ell}} \beta_{\ell, n} \sin (\pi k n)\right] .
$$

This structure of the divergent part solves the pole cancellation condition explicitly. In fact, using (4.7), we find that the divergence coming from

$$
\left(\mu_{\mathrm{eff}} \widetilde{b}_{\ell}(k)+\widetilde{c}_{\ell}(k)\right) e^{-2 \ell \mu_{\mathrm{eff}}}+d_{m}(k) e^{-4 m \mu_{\mathrm{eff}} / k},
$$

at $k=2 m / \ell$ cancels out.

Besides, we find that the remaining finite part of $\widetilde{J}^{(\mathrm{np})}\left(k, \mu_{\mathrm{eff}}\right) \equiv J\left(k, \mu\left(\mu_{\mathrm{eff}}\right)\right)-$ $J^{\text {(pert) }}\left(k, \mu_{\text {eff }}\right)$ at integers $k$ is given by

$$
\begin{gathered}
\widetilde{J}^{(\mathrm{np})}\left(k_{\mathrm{odd}}, \mu_{\mathrm{eff}}\right)=\sum_{\ell=1}^{\infty}\left[\frac{\sum_{d \mid \ell}\left(d^{3} n_{d}^{0}\right)}{16 \pi^{2} \ell^{3} k}\left(1+4 \ell \mu_{\mathrm{eff}}+8\left(\ell \mu_{\mathrm{eff}}\right)^{2}\right)\right. \\
\left.\quad+\sum_{n} \frac{(-1)^{n} k n \beta_{2 \ell, n}}{4}\right](-1)^{\ell} e^{-4 \ell \mu_{\mathrm{eff}}}+\sum_{m=1}^{\infty} h(k, m)\left[n_{d}^{g}\right] e^{-4 m \mu_{\mathrm{eff}} / k}, \\
\widetilde{J}^{(\mathrm{np})}\left(k_{\mathrm{even}}, \mu_{\mathrm{eff}}\right)=\sum_{\ell=1}^{\infty}\left[\frac{\sum_{d \mid \ell}\left(d^{3} n_{d}^{0}\right)}{2 \pi^{2} \ell^{3} k}\left(1+2 \ell \mu_{\mathrm{eff}}+2\left(\ell \mu_{\mathrm{eff}}\right)^{2}\right)+\sum_{n} \frac{k n \beta_{\ell, n}}{4}\right](-1)^{k \ell / 2} e^{-2 \ell \mu_{\mathrm{eff}}} \\
\quad+\sum_{m=1}^{\infty} h(k, m)\left[n_{d}^{g}\right] e^{-4 m \mu_{\mathrm{eff}} / k},
\end{gathered}
$$

where $h(k, m)\left[n_{d}^{g}\right]$ is a linear functional of $n_{d}^{g}$ with complicated coefficients. Note especially that the $\beta$ dependence at even integers $k$ is a simple sum, while that at odd integers $k$ is an alternating sum. This means the finite parts for integers $k$ give only two conditions.

Moreover, the condition for even integers is essentially the same as the condition from the next-to-leading WKB coefficient of $\widetilde{b}_{\ell}(k)$. Let us see this fact in more detail. We notice that the leading and the next-to-leading WKB coefficients of $\widetilde{b}_{\ell}(k)$ are related to the $g=0$ $\mathrm{GV}$ invariants $n_{d}^{0}$ and the coefficient $\beta_{\ell, n}$ through

$$
\widetilde{b}_{\ell}^{(0)}=-\frac{1}{\ell^{2} \pi^{2}} \sum_{d \mid \ell} d^{3} n_{d}^{0}, \quad \widetilde{b}_{\ell}^{(1)}=\sum_{d \mid \ell}\left(\frac{d}{12}+\frac{d^{3}}{8}\right) n_{d}^{0}-\frac{\ell}{2} \sum_{n} n \beta_{\ell, n},
$$

where the WKB expansion of $\widetilde{b}_{\ell}(k)$ is defined by

$$
\widetilde{b}_{\ell}(k)=\frac{1}{k} \sum_{m=0}^{\infty} \widetilde{b}_{\ell}^{(m)} k^{2 m}
$$

Recalling that the leading and the next-to-leading WKB coefficients of $a_{\ell}(k), b_{\ell}(k)$ and $c_{\ell}(k)$ are easily computed from the functions $J_{0}(\mu)$ and $J_{1}(\mu)$ in [9], one can compute $\widetilde{b}_{\ell}^{(0)}$ and $\widetilde{b}_{\ell}^{(1)}$ for any $\ell$. This means that one can fix $n_{d}^{0}$ and $\sum_{n} n \beta_{\ell, n}$ for any desired $d$ and $\ell$ order by order by solving (4.13). 
We also note that the non-perturbative corrections to the grand potential at $k=1,2$ are particularly simple because the contributions for $g>1$ in $J^{\mathrm{WS}}$ trivially vanish,

$$
\begin{aligned}
& \widetilde{J}^{(\mathrm{np})}\left(1, \mu_{\mathrm{eff}}\right)=\sum_{\ell=1}^{\infty}\left[\frac{\sum_{d \mid \ell}\left(d^{3} n_{d}^{0}\right)}{16 \ell}\left(\frac{8\left(\ell \mu_{\mathrm{eff}}\right)^{2}+4 \ell \mu_{\mathrm{eff}}+1}{\ell^{2} \pi^{2}}-\frac{1}{2}\right)\right. \\
& +\frac{\sum_{d \mid \ell} d\left(n_{d}^{0}-n_{2 d}^{0}\left(1-(-1)^{\frac{\ell}{d}}\right)\right)}{16 \ell} \\
& \left.+\frac{\sum_{d \mid \ell}\left(d n_{d}^{1}\right)}{\ell}+\sum_{n} \frac{(-1)^{n} n \beta_{2 \ell, n}}{4}\right](-1)^{\ell} e^{-4 \ell \mu_{\mathrm{eff}}}, \\
& \widetilde{J}^{(\mathrm{np})}\left(2, \mu_{\mathrm{eff}}\right)=\sum_{\ell=1}^{\infty}\left[\frac{\sum_{d \mid \ell}\left(d^{3} n_{d}^{0}\right)}{4 \ell}\left(\frac{2\left(\ell \mu_{\mathrm{eff}}\right)^{2}+2 \ell \mu_{\mathrm{eff}}+1}{\ell^{2} \pi^{2}}-\frac{1}{2}\right)\right. \\
& \left.+\frac{\sum_{d \mid \ell}\left(d n_{d}^{1}\right)}{\ell}+\sum_{n} \frac{n \beta_{\ell, n}}{2}\right](-1)^{\ell} e^{-2 \ell \mu_{\mathrm{eff}}} .
\end{aligned}
$$

Finally, let us study the redefinition of $\mu_{\text {eff }}$ itself. We can also express $\mu$ in terms of $\mu_{\text {eff }}$ :

$$
\mu=\mu_{\mathrm{eff}}+\frac{1}{C(k)} \sum_{\ell=1}^{\infty} e_{\ell}(k) e^{-2 \ell \mu_{\mathrm{eff}}},
$$

where the coefficients $e_{\ell}(k)$ are related to $a_{\ell}(k)$ and fixed order by order,

$$
\begin{aligned}
& e_{1}(k)=\frac{4}{\pi^{2} k} \cos \left(\frac{\pi k}{2}\right), \\
& e_{2}(k)=\frac{2}{\pi^{2} k} \cos (\pi k), \\
& e_{3}(k)=\frac{8}{3 \pi^{2} k} \cos \left(\frac{3 \pi k}{2}\right)(2+3 \cos (\pi k)), \\
& e_{4}(k)=\frac{1}{\pi^{2} k} \cos (2 \pi k)(17+32 \cos (\pi k)+16 \cos (2 \pi k)), \\
& e_{5}(k)=\frac{4}{5 \pi^{2} k} \cos \left(\frac{5 \pi k}{2}\right)(101+190 \cos (\pi k)+140 \cos (2 \pi k)+60 \cos (3 \pi k)+10 \cos (4 \pi k)) .
\end{aligned}
$$

These look simpler than $a_{\ell}(k)$.

\section{$5 \quad$ Higher membrane instanton corrections}

In this section, we would like to find the higher membrane instanton corrections $f_{\ell, 0}(k, \mu)=$ $a_{\ell}(k) \mu^{2}+b_{\ell}(k) \mu+c_{\ell}(k)$. In [15], the explicit forms of $a_{\ell}(k)$ up to $\ell=5$ have been proposed. Here we first conjecture $b_{3}(k)$ and $c_{3}(k)$, then we will further proceed to discussing $b_{4}(k)$ and $c_{4}(k)$. 


\subsection{Conjecture for $b_{3}(k)$ and $c_{3}(k)$}

Let us consider the $e^{-6 \mu}$ terms. For odd $k=2 n+1$, such terms should vanish. These contributions come from $(3,0)$ and $(1,2 n+1)$. This means that $f_{3,0}$ should behave, in the limit $k \rightarrow 2 n+1$, as

$$
\begin{aligned}
\lim _{k \rightarrow 2 n+1} f_{3,0} e^{-6 \mu} & =-\lim _{k \rightarrow 2 n+1} f_{1,2 n+1} e^{-\left(2+\frac{4(2 n+1)}{k}\right) \mu} \\
& =(-1)^{n}\left[\frac{2 n+1}{\pi(k-2 n-1)}+\frac{4 \mu+1}{\pi}\right] e^{-6 \mu} .
\end{aligned}
$$

This is consistent with $a_{3}(2 n+1)=0$, and give the conditions for $b_{3}(k)$ and $c_{3}(k)$,

$$
\begin{aligned}
& \lim _{k \rightarrow 2 n+1} b_{3}(k)=(-1)^{n} \frac{4}{\pi}, \\
& \lim _{k \rightarrow 2 n+1} c_{3}(k)=(-1)^{n}\left[\frac{2 n+1}{\pi(k-2 n-1)}+\frac{1}{\pi}\right] .
\end{aligned}
$$

Next let us consider $k=2 n / 3(n \notin 3 \mathbb{Z})$. In this case, the $\mathcal{O}\left(e^{-6 \mu}\right)$ terms come from $(3,0)$ and $(0, n)$. The pole structure of $f_{0, n} e^{-4 n \mu / k}$ takes the following form,

$$
\lim _{k \rightarrow 2 n / 3} f_{0, n} e^{-\frac{4 n}{k} \mu}=(-1)^{n-1}\left[\frac{4 n}{81 \pi^{2}\left(k-\frac{2 n}{3}\right)^{2}}+\frac{4(3 \mu+1)}{27 \pi^{2}\left(k-\frac{2 n}{3}\right)}+\frac{2 \mu^{2}+2 \mu / 3+1 / 9}{n \pi^{2}}+w_{n}\right],
$$

where

$$
w_{1}=\frac{1}{3}, \quad w_{2}=\frac{7}{6}, \quad w_{4}=\frac{187}{12}, \cdots .
$$

These poles must be canceled by the poles from $b_{3}(k)$ and $c_{3}(k)$. Thus we require the conditions at $k=2 n / 3(n \notin 3 \mathbb{Z})$,

$$
\begin{aligned}
& \lim _{k \rightarrow 2 n / 3} b_{3}(k)=(-1)^{n} \frac{4}{9 \pi^{2}\left(k-\frac{2 n}{3}\right)}+\mathcal{O}(1), \\
& \lim _{k \rightarrow 2 n / 3} c_{3}(k)=(-1)^{n}\left[\frac{4 n}{81 \pi^{2}\left(k-\frac{2 n}{3}\right)^{2}}+\frac{4}{27 \pi^{2}\left(k-\frac{2 n}{3}\right)}+\mathcal{O}(1)\right] .
\end{aligned}
$$

Finally let us consider $k=2 n$. For such $k,(3,0),(2, n),(1,2 n)$ and $(0,3 n)$ bound states contribute to the $\mathcal{O}\left(e^{-6 \mu}\right)$ terms. Since we have already known the analytic expressions of $f_{2, n}, f_{1,2 n}$ and $f_{0,3 n}$ for small $n$, we find the pole structure of each term at $k=2 n$,

$$
\begin{aligned}
& \lim _{k \rightarrow 2 n} f_{2, n} e^{-\left(4+\frac{4 n}{k}\right) \mu} \\
& \quad=(-1)^{n-1}\left[\frac{104 n}{\pi^{2}(k-2 n)^{2}}+\frac{4(26 \mu+9)}{\pi^{2}(k-2 n)}+\frac{4\left(13 \mu^{2}-4 \mu\right)}{n \pi^{2}}+x_{n}\right] e^{-6 \mu}, \\
& \lim _{k \rightarrow 2 n} f_{1,2 n} e^{-\left(2+\frac{8 n}{k}\right) \mu} \\
& =(-1)^{n-1}\left[\frac{36 n}{\pi^{2}(k-2 n)^{2}}+\frac{18(4 \mu+1)}{\pi^{2}(k-2 n)}+\frac{72 \mu^{2}}{n \pi^{2}}+y_{n}\right] e^{-6 \mu},
\end{aligned}
$$




$$
\begin{aligned}
& \lim _{k \rightarrow 2 n} f_{0,3 n} e^{-\frac{12 n}{k} \mu} \\
& \quad=(-1)^{n-1}\left[\frac{328 n}{27 \pi^{2}(k-2 n)^{2}}+\frac{328(3 \mu+1)}{27 \pi^{2}(k-2 n)}+\frac{2\left(738 \mu^{2}+246 \mu+41\right)}{27 n \pi^{2}}+z_{n}\right] e^{-6 \mu}
\end{aligned}
$$

with some constants $x_{n}, y_{n}$ and $z_{n}$. The requirement of the pole cancellation gives the conditions

$$
\begin{aligned}
& \lim _{k \rightarrow 2 n} b_{3}(k)=(-1)^{n}\left[\frac{1912}{9 \pi^{2}(k-2 n)}+\mathcal{O}(1)\right] \\
& \lim _{k \rightarrow 2 n} c_{3}(k)=(-1)^{n}\left[\frac{4108 n}{27 \pi^{2}(k-2 n)^{2}}+\frac{1786}{27 \pi^{2}(k-2 n)}+\mathcal{O}(1)\right] .
\end{aligned}
$$

(5.2), (5.5) and (5.7) are the pole conditions that $b_{3}(k)$ and $c_{3}(k)$ should satisfy.

If we believe the relation (4.7) holds for any $\ell$, the whole system of $b_{\ell}(k)$ and $c_{\ell}(k)$ are determined explicitly by $\widetilde{b}_{\ell}(k)$, which consists of fewer coefficients. To determine $\widetilde{b}_{3}(k)$, we make an ansatz for $\widetilde{b}_{3}(k)$ similar to the form of $a_{3}(k)$, as in the case for $\ell=1,2$. Here we propose the explicit form of $\widetilde{b}_{3}(k)$ satisfying both the above pole structures and the correct WKB expansions in [15],

$$
\widetilde{b}_{3}(k)=\frac{4}{3 \pi} \cot \left(\frac{3 \pi k}{2}\right) \cos \left(\frac{\pi k}{2}\right)(13+19 \cos (\pi k)+9 \cos (2 \pi k)) .
$$

Using (4.7), $b_{3}(k)$ and $c_{3}(k)$ can be reconstructed from $\widetilde{b}_{3}(k)$ as

$$
\begin{aligned}
b_{3}(k)= & \frac{4}{\pi^{2} k}\left[13 \cos \left(\frac{\pi k}{2}\right)+5 \cos \left(\frac{3 \pi k}{2}\right)\right] \\
& +\frac{1}{3 \pi} \csc \left(\frac{3 \pi k}{2}\right)(241+405 \cos (\pi k)+222 \cos (2 \pi k)+79 \cos (3 \pi k)+9 \cos (4 \pi k)), \\
c_{3}(k)= & \left(\frac{\pi^{2}}{6}+\frac{\pi^{2} k^{2}}{48}\right) a_{3}(k)+\frac{\pi^{4} k^{2}}{12} a_{1}(k)^{3}-\frac{1}{2} k \sec \left(\frac{\pi k}{2}\right)-\frac{6}{\pi} \cot \left(\frac{\pi k}{2}\right) \cos \left(\frac{\pi k}{2}\right) \\
& +\frac{1}{\pi} \cot \left(\frac{3 \pi k}{2}\right)\left[\cos \left(\frac{\pi k}{2}\right)+\frac{10}{9} \cos \left(\frac{3 \pi k}{2}\right)+\cos \left(\frac{5 \pi k}{2}\right)\right] \\
& +k\left[3 \cos \left(\frac{\pi k}{2}\right)+\frac{79}{6} \cos \left(\frac{3 \pi k}{2}\right)+\frac{5}{2} \cos \left(\frac{5 \pi k}{2}\right)\right] \\
& +k \csc ^{2}\left(\frac{3 \pi k}{2}\right)\left[95 \cos \left(\frac{\pi k}{2}\right)+\frac{343}{6} \cos \left(\frac{3 \pi k}{2}\right)+19 \cos \left(\frac{5 \pi k}{2}\right)\right] .
\end{aligned}
$$

Note that $\widetilde{b}_{3}(k)$ is much simpler than $b_{3}(k)$ and $c_{3}(k)$. One can also check that these results reproduce the coefficients of $e^{-6 \mu}$ for $k=2,4$ correctly,

$$
\begin{aligned}
\lim _{k \rightarrow 2} \sum_{m=0}^{3} f_{3-m, m} e^{-\left(2(3-m)+\frac{4 m}{k}\right) \mu} & =\left[\frac{736 \mu^{2}-304 \mu / 3+154 / 9}{3 \pi^{2}}-32\right] e^{-6 \mu}, \\
\lim _{k \rightarrow 4} \sum_{m=0}^{3} f_{3-m, 2 m} e^{-\left(2(3-m)+\frac{8 m}{k}\right) \mu} & =\left[-\frac{736 \mu^{2}-304 \mu / 3+154 / 9}{6 \pi^{2}}+32\right] e^{-6 \mu} .
\end{aligned}
$$


As mentioned in the previous section, (5.8) can be recast into the form

$$
\widetilde{b}_{3}(k)=-\frac{3}{2 \pi} \cos \left(\frac{3 \pi k}{2}\right)\left[n_{3}^{0} \cot \left(\frac{\pi k}{2}\right)+\frac{n_{1}^{0}}{3^{2}} \cot \left(\frac{3 \pi k}{2}\right)+8 \sin (\pi k)\right],
$$

which is consistent with our general formula in (4.10).

\subsection{Further conjecture for $b_{4}(k)$ and $c_{4}(k)$}

Let us proceed to the 4 -membrane instanton coefficients. To determine $\widetilde{b}_{4}(k)$, we make an ansatz for $\widetilde{b}_{4}(k)$ similar to the form of $a_{4}(k)$. If we require that, in the sectors with

- $(4,0)$ and $(0, n)$ bound states at $k=n / 2$ (for $n=1,3,5,7)$,

- $(4,0),(2, n)$ and $(0,2 n)$ bound states at $k=n$ (for $n=1,3$ ),

- $(4,0),(3,1),(2,2),(1,3)$ and $(0,4)$ bound states at $k=2$,

the poles cancel and the finite terms for integer $k$ match with our fitting results (A.1), we find explicitly that

$$
\widetilde{b}_{4}(k)=\frac{1}{2 \pi} \cot (2 \pi k)(164+288 \cos (\pi k)+197 \cos (2 \pi k)+96 \cos (3 \pi k)+32 \cos (4 \pi k)) .
$$

Note that as we have studied in $(4.10), \widetilde{b}_{4}(k)$ can also be put into the form

$$
\widetilde{b}_{4}(k)=-\frac{2}{\pi} \cos (2 \pi k)\left[n_{4}^{0} \cot \left(\frac{\pi k}{2}\right)+\frac{n_{2}^{0}}{2^{2}} \cot (\pi k)+\frac{n_{1}^{0}}{4^{2}} \cot (2 \pi k)+48 \sin (\pi k)+16 \sin (2 \pi k)\right] .
$$

From this, we can reconstruct $b_{4}(k)$ and $c_{4}(k)$ without difficulty. Since the expressions are lengthy, we shall list here only the expansion around $k=0$,

$$
\begin{aligned}
b_{4}(k)= & \frac{33635}{12 \pi^{2} k}-\frac{17165 k}{6}+\frac{127339 \pi^{2} k^{3}}{90}-\frac{12223 \pi^{4} k^{5}}{27}+\frac{1945973 \pi^{6} k^{7}}{18900}+\mathcal{O}\left(k^{9}\right), \\
c_{4}(k)= & \left(-\frac{1225}{6}+\frac{54113}{144 \pi^{2}}\right) \frac{1}{k}+\left(\frac{32821}{144}+175 \pi^{2}\right) k+\left(-\frac{57512 \pi^{2}}{135}-\frac{217 \pi^{4}}{3}\right) k^{3} \\
& +\left(\frac{5405089 \pi^{4}}{22680}+\frac{175 \pi^{6}}{9}\right) k^{5}+\left(-\frac{5057377 \pi^{6}}{64800}-\frac{1343 \pi^{8}}{360}\right) k^{7}+\mathcal{O}\left(k^{9}\right) .
\end{aligned}
$$

The first two coefficients are found to match the results of [9] correctly. However, these matches are automatic from the above cancellation mechanism. ${ }^{3}$

We can repeat the same analysis for $b_{5}(k)$ and $c_{5}(k)$. However, in an ansatz of $\widetilde{b}_{5}(k)$ similar to $a_{5}(k)$, there are more coefficients than conditions and we cannot determine them explicitly. One way to see it is that, as we noted below (4.12), the finite values of $J(k, \mu)$ for integers $k$ gives two conditions to determine two coefficients $\beta_{4, n}$ in $\widetilde{b}_{4}(k)$, while $\widetilde{b}_{5}(k)$ is expected to have more coefficients which cannot be determined only by one condition in (A.1).

\footnotetext{
${ }^{3}$ We thank the authors of [15] for checking that the higher coefficients in (5.14) also perfectly agree with the ones obtained from the WKB expansion in [15].
} 


\section{Summary and discussions}

In this paper, we have studied the non-perturbative corrections to the ABJM partition function in the Fermi-gas approach. These non-perturbative corrections contain the contributions from the bound states of the worldsheet instantons and the membrane instantons, which originate from M2-branes wrapping two different cycles $\mathcal{M}$ and $\mathcal{W}$ on the gravity dual side.

We have proposed an expression for the coefficients of the bound states $f_{\ell, m}(k, \mu)$ and the 3- and 4-membrane instanton corrections explicitly. We have found that the summation over the bound states is beautifully incorporated into the worldsheet instanton correction by redefining the chemical potential $\mu$. We believe that our results on the bound states open up a new era in understanding instanton effects in M-theory since, unlike the worldsheet instanton or the membrane instanton, the bound states were not accessible from any established methods such as the topological strings or the WKB analysis in the Fermi-gas. Our method is basically the same as our previous work [18] by requiring the singularities coming from each instanton contribution to cancel and matching the finite results and the WKB expansions. We have also found that the non-perturbative correction to the grand potential can be reexpressed in a simpler form by using the redefined chemical potential. This result implies a deep hidden structure in the ABJM partition function.

We shall conclude our paper with discussions on the further directions.

Firstly, we have only used a limited number of data to propose analytic expressions, which are considered to have infinite coefficients potentially. It would be important to check our results in more details by increasing the data such as the WKB expansions and the finite results in the grand potential.

Secondly, after determining the coefficients up to 4-membrane instanton, we would like to head for a more general structure of the membrane instanton correction. For example, we have found that the redefinition of the chemical potential simplifies the expression substantially and the new expression enjoys an interesting relation among the coefficients. It would be interesting to understand the physical meaning of the redefinition. Perhaps, $\mu_{\text {eff }}$ might be interpreted as a certain generalization of the flat coordinate on the quantum moduli space of local $\mathbb{F}_{0}$, including the membrane instanton corrections. The similarity pointed out below (3.30) may be useful to clarify the interpretation of $\mu_{\text {eff }}$. Along this line, we would like to raise a question whether $\mu$ or $\mu_{\text {eff }}$ is more "fundamental". As we have seen, most of our general arguments are expressed more simply in $\mu_{\text {eff }}$. However, though we observed [18] in (A.1) a similarity in coefficients between $J(1, \mu)$ and $J(2, \mu)$ and between $J(3, \mu)$ and $J(6, \mu)$ even for terms not multiplied by $1 / \pi^{2}$, this similarity is not found when expressed in $\mu_{\text {eff }}$.

Also, our general expression (4.10) for $\widetilde{b}_{\ell}(k)$, guided by the pole cancellation mechanism, contains the coefficients $\beta_{\ell, n}$ and the relation between $\mu$ and $\mu_{\text {eff contains the co- }}$ efficients in $e_{\ell}(k)$ or $a_{\ell}(k)$. It is interesting to see whether these coefficients can also be expressed in terms of the GV invariants. On one hand, since no other topological invariants are known so far besides the GV invariants and the GV invariants are derived from M-theory, one tends to expect these coefficients are also the GV invariants in disguise. On 
the other hand, since the GV invariant is counting the holomorphic cycles, it seems also natural to expect the information of the membrane instanton is encoded by different invariants counting the Lagrangian submanifolds. It is important to study higher membrane instanton effects to increase more coefficients to find out the structure.

Finally, it would be also interesting to study the relation, if any, between the worldsheet instanton corrections and the membrane instanton corrections under the inversion of the coupling $k / 2 \rightarrow 2 / k$ along the lines of [24].

\section{Acknowledgments}

We are grateful to Shinji Hirano, Kazuo Hosomichi, Hiroaki Kanno, Soo-Jong Rey, Kazuhiro Sakai, Masaki Shigemori, Seiji Terashima, and especially Flavio Calvo and Marcos Marino, for useful discussions. S.M. is grateful to Yukawa institute for theoretical physics for hospitality, where part of this work was done. The work of Y.H. is supported in part by the JSPS Research Fellowship for Young Scientists, while the work of K.O. is supported in part by JSPS Grant-in-Aid for Young Scientists (B) \#23740178.

\section{A $\quad J^{(\mathrm{np})}(k, \mu)$ at $k=1,2,3,4,6$}

Here we summarize the non-perturbative corrections to the grand potential at $k=$ $1,2,3,4,6$. These are the updated data, part of which are previously listed in [18]. These coefficients can be fixed by the numerical fitting from the exact values of the partition function. See [18] for more detail:

$$
\begin{aligned}
J^{(\mathrm{np})}(1, \mu)= & {\left[\frac{4 \mu^{2}+\mu+1 / 4}{\pi^{2}}\right] e^{-4 \mu}+\left[-\frac{52 \mu^{2}+\mu / 2+9 / 16}{2 \pi^{2}}+2\right] e^{-8 \mu} } \\
& +\left[\frac{736 \mu^{2}-152 \mu / 3+77 / 18}{3 \pi^{2}}-32\right] e^{-12 \mu} \\
& +\left[-\frac{2701 \mu^{2}-13949 \mu / 48+11291 / 768}{\pi^{2}}+466\right] e^{-16 \mu} \\
& +\left[\frac{161824 \mu^{2}-317122 \mu / 15+285253 / 300}{5 \pi^{2}}-6720\right] e^{-20 \mu} \\
& +\left[-\frac{1227440 \mu^{2}-2686522 \mu / 15+631257 / 80}{3 \pi^{2}}-\frac{292064}{3}\right] e^{-24 \mu}+\mathcal{O}\left(e^{-28 \mu}\right), \\
J^{(\mathrm{np})}(2, \mu)= & {\left[\frac{4 \mu^{2}+2 \mu+1}{\left.\pi^{2}\right] e^{-2 \mu}+\left[-\frac{52 \mu^{2}+\mu+9 / 4}{2 \pi^{2}}+2\right] e^{-4 \mu}}\right.} \\
& +\left[\frac{736 \mu^{2}-304 \mu / 3+154 / 9}{3 \pi^{2}}-32\right] e^{-6 \mu} \\
& +\left[-\frac{2701 \mu^{2}-13949 \mu / 24+11291 / 192}{\pi^{2}}+466\right] e^{-8 \mu} \\
& +\left[\frac{161824 \mu^{2}-634244 \mu / 15+285253 / 75}{5 \pi^{2}}-6720\right] e^{-10 \mu} \\
& +\left[-\frac{1227440 \mu^{2}-5373044 \mu / 15+631257 / 20}{3 \pi^{2}}-\frac{292064}{3}\right] e^{-12 \mu}+\mathcal{O}\left(e^{-14 \mu}\right),
\end{aligned}
$$




$$
\begin{aligned}
J^{(\mathrm{np})}(3, \mu)= & \frac{4}{3} e^{-\frac{4}{3} \mu}-2 e^{-\frac{8}{3} \mu}+\left[\frac{4 \mu^{2}+\mu+1 / 4}{3 \pi^{2}}+\frac{20}{9}\right] e^{-4 \mu}-\frac{88}{9} e^{-\frac{16}{3} \mu}+\frac{108}{5} e^{-\frac{20}{3} \mu} \\
& +\left[-\frac{52 \mu^{2}+\mu / 2+9 / 16}{6 \pi^{2}}-\frac{298}{9}\right] e^{-8 \mu}+\frac{25208}{189} e^{-\frac{28}{3} \mu}+\mathcal{O}\left(e^{-\frac{32}{3} \mu}\right), \\
J^{(\mathrm{np})}(4, \mu)= & e^{-\mu}+\left[-\frac{4 \mu^{2}+2 \mu+1}{2 \pi^{2}}\right] e^{-2 \mu}+\frac{16}{3} e^{-3 \mu}+\left[-\frac{52 \mu^{2}+\mu+9 / 4}{4 \pi^{2}}+2\right] e^{-4 \mu} \\
& +\frac{256}{5} e^{-5 \mu}+\left[-\frac{736 \mu^{2}-304 \mu / 3+154 / 9}{6 \pi^{2}}+32\right] e^{-6 \mu}+\frac{4096}{7} e^{-7 \mu}+\mathcal{O}\left(e^{-8 \mu}\right), \\
J^{(\mathrm{np})}(6, \mu)= & \frac{4}{3} e^{-\frac{2}{3} \mu}-2 e^{-\frac{4}{3} \mu}+\left[\frac{4 \mu^{2}+2 \mu+1}{3 \pi^{2}}+\frac{20}{9}\right] e^{-2 \mu}-\frac{88}{9} e^{-\frac{8}{3} \mu}+\frac{108}{5} e^{-\frac{10}{3} \mu} \\
& +\left[-\frac{52 \mu^{2}+\mu+9 / 4}{6 \pi^{2}}-\frac{298}{9}\right] e^{-4 \mu}+\frac{25208}{189} e^{-\frac{14}{3} \mu}+\mathcal{O}\left(e^{-\frac{16}{3} \mu}\right) .
\end{aligned}
$$

\section{B Known results of membrane instanton corrections}

Here we summarize various known results of the membrane instanton coefficients. The coefficients of 1-membrane instanton are given by

$$
\begin{aligned}
& a_{1}(k)=-\frac{4}{\pi^{2} k} \cos \left(\frac{\pi k}{2}\right), \\
& b_{1}(k)=\frac{2}{\pi} \csc \left(\frac{\pi k}{2}\right) \cos ^{2}\left(\frac{\pi k}{2}\right), \\
& c_{1}(k)=\left[-\frac{2}{3 k}+\frac{5 k}{12}+\frac{1}{\pi} \cot \left(\frac{\pi k}{2}\right)+\frac{k}{2} \csc ^{2}\left(\frac{\pi k}{2}\right)\right] \cos \left(\frac{\pi k}{2}\right),
\end{aligned}
$$

while those of 2-membrane instanton are

$$
\begin{aligned}
a_{2}(k)= & -\frac{2}{\pi^{2} k}(4+5 \cos (\pi k)), \\
b_{2}(k)= & \frac{4}{\pi^{2} k}(1+\cos (\pi k))+\frac{1}{\pi} \csc (\pi k)(2+3 \cos (\pi k))^{2}, \\
c_{2}(k)= & \left(-\frac{1}{3 k}+\frac{1}{4 \pi} \cot (\pi k)\right)(4+5 \cos (\pi k))+\frac{7 k}{24}(7 \cos (\pi k)-4) \\
& +\frac{k}{4} \csc ^{2}(\pi k)(20+21 \cos (\pi k)) .
\end{aligned}
$$

The coefficients $a_{\ell}(k)$ are determined up to $\ell=5$ as

$$
\begin{aligned}
a_{3}(k)= & -\frac{8}{3 \pi^{2} k} \cos \left(\frac{\pi k}{2}\right)(19+28 \cos (\pi k)+3 \cos (2 \pi k)), \\
a_{4}(k)=- & \frac{1}{\pi^{2} k}(364+560 \cos (\pi k)+245 \cos (2 \pi k)+48 \cos (3 \pi k)+8 \cos (4 \pi k)), \\
a_{5}(k)=- & \frac{8}{5 \pi^{2} k} \cos \left(\frac{\pi k}{2}\right)(2113+3374 \cos (\pi k)+1751 \cos (2 \pi k)+525 \cos (3 \pi k) \\
& +145 \cos (4 \pi k)+25 \cos (5 \pi k)+5 \cos (6 \pi k)) .
\end{aligned}
$$




\section{Relation of $\mu$ and $\mu_{\text {eff }}$ for even $k$}

In this appendix, we derive (3.29) for even integer $k=2 n$. Our goal here is to exactly perform the sum

$$
\sum_{\ell=1}^{\infty} a_{\ell}(2 n) e^{-2 \ell \mu} .
$$

Since $k a_{\ell}(k)$ is a periodic function of $k$, the values of $a_{\ell}(2 n)$ are related to the leading WKB coefficient of $a_{\ell}(k)$ :

$$
a_{\ell}(2 n)=(-1)^{\ell n} \frac{a_{\ell}^{(0)}}{2 n}
$$

where the WKB expansion is given by

$$
a_{\ell}(k)=\frac{1}{k} \sum_{m=0}^{\infty} a_{\ell}^{(m)} k^{2 m}
$$

We find that $a_{\ell}^{(0)}$ is generically given by

$$
a_{\ell}^{(0)}=-\frac{4^{\ell}}{\ell \pi^{2}}\left[\frac{(2 \ell-1) ! !}{\ell !}\right]^{2} .
$$

This result can be confirmed by using the large $\mu$ expansion of $J_{0}(\mu)$ in [9],

$$
\begin{aligned}
J_{0}(\mu) & =\frac{e^{\mu}}{4}{ }_{3} F_{2}\left(\frac{1}{2}, \frac{1}{2}, \frac{1}{2} ; 1, \frac{3}{2} ; \frac{e^{2 \mu}}{16}\right)-\frac{e^{2 \mu}}{8 \pi^{2}}{ }_{4} F_{3}\left(1,1,1,1 ; \frac{3}{2}, \frac{3}{2}, 2 ; \frac{e^{2 \mu}}{16}\right) \\
& =\frac{2 \mu^{3}}{3 \pi^{2}}+\frac{\mu}{3}+\frac{2 \zeta(3)}{\pi^{2}}+\sum_{\ell=1}^{\infty}\left(a_{\ell}^{(0)} \mu^{2}+b_{\ell}^{(0)} \mu+c_{\ell}^{(0)}\right) e^{-2 \ell \mu} .
\end{aligned}
$$

Therefore we finally obtain

$$
\sum_{\ell=1}^{\infty} a_{\ell}(2 n) e^{-2 \ell \mu}=(-1)^{n-1} \frac{2}{n \pi^{2}} e^{-2 \mu}{ }_{4} F_{3}\left(1,1, \frac{3}{2}, \frac{3}{2} ; 2,2,2 ;(-1)^{n} 16 e^{-2 \mu}\right) .
$$

Open Access. This article is distributed under the terms of the Creative Commons Attribution License which permits any use, distribution and reproduction in any medium, provided the original author(s) and source are credited.

\section{References}

[1] O. Aharony, O. Bergman, D.L. Jafferis and J. Maldacena, $N=6$ superconformal Chern-Simons-matter theories, M2-branes and their gravity duals, JHEP 10 (2008) 091 [arXiv:0806.1218] [INSPIRE].

[2] A. Kapustin, B. Willett and I. Yaakov, Exact results for Wilson loops in superconformal Chern-Simons theories with matter, JHEP 03 (2010) 089 [arXiv:0909.4559] [INSPIRE]. 
[3] D.L. Jafferis, The exact superconformal R-symmetry extremizes Z, JHEP 05 (2012) 159 [arXiv: 1012.3210] [INSPIRE].

[4] N. Hama, K. Hosomichi and S. Lee, Notes on SUSY gauge theories on three-sphere, JHEP 03 (2011) 127 [arXiv: 1012.3512] [INSPIRE].

[5] V. Pestun, Localization of gauge theory on a four-sphere and supersymmetric Wilson loops, Commun. Math. Phys. 313 (2012) 71 [arXiv:0712.2824] [INSPIRE].

[6] N. Drukker, M. Mariño and P. Putrov, From weak to strong coupling in ABJM theory, Commun. Math. Phys. 306 (2011) 511 [arXiv:1007.3837] [INSPIRE].

[7] I.R. Klebanov and A.A. Tseytlin, Entropy of near extremal black p-branes, Nucl. Phys. B 475 (1996) 164 [hep-th/9604089] [INSPIRE].

[8] H. Fuji, S. Hirano and S. Moriyama, Summing up all genus free energy of ABJM matrix model, JHEP 08 (2011) 001 [arXiv:1106.4631] [INSPIRE].

[9] M. Mariño and P. Putrov, ABJM theory as a Fermi gas, J. Stat. Mech. 1203 (2012) P03001 [arXiv: 1110.4066] [INSPIRE].

[10] K. Okuyama, A note on the partition function of ABJM theory on $S^{3}$, Prog. Theor. Phys. 127 (2012) 229 [arXiv:1110.3555] [InSPIRE].

[11] M. Mariño and P. Putrov, Interacting fermions and $N=2$ Chern-Simons-matter theories, arXiv: 1206.6346 [INSPIRE].

[12] A. Klemm, M. Mariño, M. Schiereck and M. Soroush, ABJM Wilson loops in the Fermi gas approach, arXiv:1207.0611 [INSPIRE].

[13] H. Awata, S. Hirano and M. Shigemori, The partition function of ABJ theory, arXiv: 1212.2966 [INSPIRE].

[14] O. Aharony, O. Bergman and D.L. Jafferis, Fractional M2-branes, JHEP 11 (2008) 043 [arXiv: 0807.4924] [INSPIRE].

[15] F. Calvo and M. Mariño, Membrane instantons from a semiclassical TBA, arXiv:1212.5118 [INSPIRE].

[16] Y. Hatsuda, S. Moriyama and K. Okuyama, Exact results on the ABJM Fermi gas, JHEP 10 (2012) 020 [arXiv:1207.4283] [INSPIRE].

[17] P. Putrov and M. Yamazaki, Exact ABJM partition function from TBA, Mod. Phys. Lett. A 27 (2012) 1250200 [arXiv:1207.5066] [INSPIRE].

[18] Y. Hatsuda, S. Moriyama and K. Okuyama, Instanton effects in ABJM theory from Fermi gas approach, JHEP 01 (2013) 158 [arXiv:1211.1251] [INSPIRE].

[19] M. Hanada et al., Numerical studies of the ABJM theory for arbitrary $N$ at arbitrary coupling constant, JHEP 05 (2012) 121 [arXiv: 1202.5300] [INSPIRE].

[20] A. Cagnazzo, D. Sorokin and L. Wulff, String instanton in $A d S_{4} \times C P^{3}$, JHEP 05 (2010) 009 [arXiv:0911.5228] [INSPIRE].

[21] N. Drukker, M. Mariño and P. Putrov, Nonperturbative aspects of ABJM theory, JHEP 11 (2011) 141 [arXiv:1103.4844] [INSPIRE].

[22] S.H. Katz, A. Klemm and C. Vafa, M theory, topological strings and spinning black holes, Adv. Theor. Math. Phys. 3 (1999) 1445 [hep-th/9910181] [InSPIRE].

[23] M. Aganagic, M. Mariño and C. Vafa, All loop topological string amplitudes from Chern-Simons theory, Commun. Math. Phys. 247 (2004) 467 [hep-th/0206164] [InSPIRE].

[24] G. Lockhart and C. Vafa, Superconformal partition functions and non-perturbative topological strings, arXiv:1210.5909 [INSPIRE]. 